\title{
On the Roots of the Confluent Hypergeometric Functions.
}

\author{
By Archd. Milnk, Research Student, Edinburgh University \\ Mathematical Laboratory.
}

(Read 19th February 1915. Received 3rd March 1915).

\section{$\$ 1$. Contents of Paper.}

In the present paper the disposition of the roots of the confluent hypergeometric functions-denoted by $W_{k, m}(z)$ - as affected by changing the parameters $k$ and $m$ is investigated. The results are then shewn in a graphical form, and various typical illustrations of the functions are given. By giving special values to $k$ and $m$ it is then exemplified how the roots of other functions expressible in terms of $W_{k, m}(z)$ may be studied. The zeros of the parabolic cylinder functions are then discussed. Some of the properties of an allied class of functions, denoted by $\psi_{n}(z)$, are then given, and finally, it is shewn how the properties of Abel's function $\phi_{m}(\approx)$ may be obtained from results already given.

\section{\$2. Definition of $W_{k, m}(z)$.}

In the Bulletin of the American Mathemalical Sisciety, 2nd Series, Vol. 10, p. 125 (1903), Whittaker discliss sd a function $W_{k, m}(z)$, which may be derived from the hypergeometric function by making the exponents at two of the singularities infinite, and then making these two singularities to coalesce, so as to have an irregular singularity at $\infty$. The function is defined for all values of $z$ (real or complex) by the equation

$$
W_{k, m}(z)=\frac{\Gamma\left(k+\frac{1}{2}-m\right)}{2 \pi} e^{-\frac{1}{2} z+\frac{1}{2} i \pi} z^{x} \int(-t)^{-k-\frac{1}{i}+m}\left(1+\frac{t}{z}\right)^{k-1+m} e^{-t} d t
$$

with a defined path of integration. This complex integral can, in certain circumstances, be replaced by the real integral

$$
W_{k, m}(z)=\frac{1}{\Gamma\left(-k+\frac{1}{2}+m\right)} z^{k} e^{-\frac{1}{2} z} \int_{0}^{\infty} t^{-k-\frac{1}{2}+m}\left(1+\frac{t}{z}\right)^{k-\frac{1}{t}+m} e^{-t} d t .
$$


Further, it was shewn that the differential equation which $W_{k, m}(z)$ satisfies is

$$
\frac{d^{2} W}{d z^{2}}+\left(-\frac{1}{4}+\frac{k}{z}+\frac{\frac{1}{4}-m^{2}}{z^{2}}\right) W=0
$$

§3. Relation between the roots of $\dot{W}_{k, m}(z)$ and $W_{k-1, m}(z)$.

If in the above equation we make the substitution

$$
W_{k, m}(z)=e^{-\frac{t}{2} z} z^{k} v
$$

we get the equation

$$
z^{2} \frac{d^{2} v}{d z^{3}}+z(2 k-z) \frac{d v}{d z}+\left\{\left(k-\frac{1}{2}\right)^{2}-m^{2}\right\} v=0 .
$$

Consider the nature of solutions at points where $\frac{d v}{d z}$ vanishes. If $\left(k-\frac{1}{2}\right)^{2}-m^{2}$ is negative, the equation at such points is of the form $\frac{d^{2} v}{d z^{2}}-P v=0$, which shews that in this case $\frac{d v}{d z}$ would vanish for no finite value of $z$; that is to say, in this instance, the curve must always be convex to the real axis, and of course, as a consequence, the function will have no zeros. If, on the other hand, $\left(k-\frac{1}{2}\right)^{2}-m^{2}$ is positive, since the equation is of the form $\frac{d^{2} v}{d z^{2}}+P v=0$, the curve may be concave to the real axis, in which event zeros of the function appear. Then since $z^{2}$ is positive for all real values of $z$, the signs of $\frac{d^{2} v}{d z^{2}}$ and of $\left\{\left(k-\frac{1}{2}\right)^{2}-m^{2}\right\} v$ are opposite at any point where $\frac{d v}{d z}$ vanishes. Let $z=\zeta_{1}$ and $z=\zeta_{2}$ be two consecutive zeros of $\frac{d v}{d z}$. Then since $\frac{d^{2} v}{d z^{2}}$ represents at any point the gradient of $\frac{d v}{d z}$, the sign of $\frac{d^{2} v}{d z^{2}}$ just after leaving $z=\zeta_{2}$ must be opposite to what it is just before $z=\zeta_{2}$. Hence the signs of $\left\{\left(k-\frac{1}{2}\right)^{2}-m^{2}\right\} v$ just after $z=\zeta_{1}$ and just before $z=\zeta_{2}$, are unlike, i.e. $\left\{\left(k-\frac{1}{2}\right)^{2}-\dot{m}^{2}\right\} v$ crosses the $z$-axis an odd number of times in the interval $\overline{\zeta_{1} \zeta_{2}}$. But these are consecutive zeros of $\frac{d v}{d z}$. Therefore, by Rolle's Theorem the $z$-axis is crossed only once in that interval, i.e. the 
roots of $\frac{d v}{d z}$ and of $\left\{\left(k-\frac{1}{2}\right)^{2}-m^{2}\right\} v$ alternate. But $v=e^{k z} z^{-k} W_{k, m}(z)$. Hence, making the necessary substitutions, we get that, apart from the root $z=0$, which may be a common root (see $\$ 4$ ), the roots of $z \frac{d W_{k, m}}{d z}+\left(\frac{1}{2} z-k\right) W_{k, m}$ and of $\left\{\left(k-\frac{1}{2}\right)^{2}-m^{2}\right\} W_{k, m}$ alternate.

If now we differentiate with respect to $z$ the equation $\frac{2 \pi}{\Gamma\left(k+\frac{1}{2}-m\right)} e^{j z+t \pi i} z^{-k} W_{k, m}(z)=\int_{\delta}(-t)^{-k-t+m}\left(1+\frac{t}{z}\right)^{k-1+m} e^{-t} d t$, we derive the recurrence-formula

$$
\left.z \frac{d W_{k, m}}{d z}+\left(\frac{1}{2} z-k\right) W_{k, m}-\left\{k-\frac{1}{2}\right)^{2}-m^{2}\right\} W_{k-1, m}=0 .
$$

Using this in our result, we obtain that, apart from the root $z=0$, which on occsision is a common root, the roots of $W_{k, m}(z)$ and of $W_{k-1, m}(z)$ alternate.

As an immediate corollary it follows that if any given value of the parameter $k$ is increased by unity while $m$ is left unaltered, then the number of roots of $W_{k, m}(z)$ is increased by unity.

We may note here that if we revert to the differential equation satisfied by $W_{k, m}(z)$, viz., $\frac{d^{2} W}{d z^{3}}+f(z) W=0$, points of inflexion on the graph of $W_{m, m}(z)$ for any given values of $k$ and $m$, will be obtained from the solution of the equation $f(z)=0$, which, being a quadratic, presents no difficulties.

Again, by definition we have

$$
W_{k, m}(z)=M z^{k} \int(-t)^{-k-1+m}\left(1+\frac{t}{z}\right)^{k-1+m} e^{-t} d t
$$

where $M$ is a multiplier involving constants and an exponential, not affecting the occurrence of the roots.

If we put $t+z=x$, this can be written

$$
W_{k, m}(z)=M^{\prime} z-m \int(x-z)^{-k-1+m} x^{k-1+m} e^{-z} d x .
$$

If now $k-\frac{1}{2}-m$ be integral, $=n$ say, we get from Cauchy's theorem for the $n^{\text {th }}$ derivative of a function

$$
\int x^{k-1+m} e^{-x}(x-z)^{-n-1} d x=\text { const. } \times \frac{d^{n}}{d z^{n}} f(z)
$$

where $f(x)=z^{k-1+m_{0}-z}$. 
Hence we have

$$
\begin{gathered}
W_{k, m}(z)=M^{\prime \prime} z^{j-m} \frac{d^{n}}{d z^{n}}\left(z^{n+2 m} e^{-z}\right) \\
=(-1)^{n} M^{\prime \prime} e^{-z} z^{-m}\left[z^{n+2 m}-{ }_{n+m} C_{1} z^{n+2 m-1}+\ldots+(-1)^{n-1}{ }_{n+m} C_{n} z^{2 m}\right] \\
=M^{\prime \prime \prime} z^{3+m}\left[z^{n}-{ }_{n+m} C_{1} z^{n-1}+\ldots+(-1)^{n-1}{ }_{n+m} C_{n}\right]
\end{gathered}
$$

where the expression inside the brackets is a polynomial since $n$ is integral. If $n$, i.e. $k-\frac{1}{2}-m$, vanishes, this expression reduces to a constant, and apart from $z=0, W_{k, m}(z)$ has no zero. If $n=1$, i.e. if $k-\frac{3}{2}-m=0$, the expression is obviously linear and there is one root. Clearly each time $n$-the degree of the polynomial-is increased by unity, i.e. $k$ is increased by unity, another root is introduced.

\section{\$4. Graphical illustrations.}

The results so far obtained may be illustrated graphically to shew the disposition of roots according to any given values of $k$ and $m$ (see Fig. 1). If we measure values of $k$ along the horizontal axis and values of $m$ on the vertical axis, and if we draw the portion of the line $k-m-\frac{1}{2}=0$ above the $k$-axis and the portion of the line $k+m-\frac{1}{2}=0$ below that axis-derived from $\left(k-\frac{1}{2}\right)^{2}-m^{2}=0$ - the plane will thereby be divided into two regions, in one of which $W_{k, m}(z)$ will have no zeros when $k$ and $m$ have uny of the values in that region. Again, since one root appears when $k-\frac{1}{2}-m=1$, and two appear when $k-\frac{1}{2}-m=2$, and so on, if we draw parallel lines at distances unity along the horizontal axis, the region shewing when roots occur is thereby divided into sections, where the numbers indicate the number of zeros that $W_{k, m}(z)$ has if the plotted values of $k$ and $m$ lie in that section. Thus, in our differential equation

$$
\frac{d^{2} y}{d z^{2}}+\left(-1+\frac{k}{z}+\frac{t-m^{2}}{z^{2}}\right) y=0
$$

the solution $W_{k, m}(z)$ will have no zeros when $k=0 \cdot 2, m=0 \cdot 1$, two zeros when $k=4 \cdot 1, m=2 \cdot 2$, and so on.

Graphs are given shewing the form of $W_{k, m}(z)$ for various values of $k$ and $m$. (See Figs. 2-7). The numerical values necessary for plotting were obtained from the formula 


$$
W_{k, m}(z)=\frac{\Gamma(-9 m)}{\Gamma\left(\frac{1}{2}-m-k\right)} M\left(z j+\frac{\Gamma(2 m)}{\Gamma\left(\frac{1}{2}+m-k\right)} P(z)\right.
$$

where

$$
\begin{aligned}
& M(z)=z^{1+m} e^{\frac{1 z}{z}}\left[1-\frac{\frac{1}{2}+m+k}{1 \cdot(2 m+1)} z+\frac{\left(\frac{1}{2}+m+k\right)\left(\frac{3}{2}+m+k\right)}{1.2 \cdot(2 m+1)(2 m+2)} z^{2}-\ldots .\right] \\
& =z^{\frac{1}{2}+m} e^{-\frac{1}{2} z}\left[1+\frac{\frac{1}{2}+m-k}{1 \cdot(2 m+1)} z+\frac{\left(\frac{1}{2}+m-k\right)\left(\frac{3}{2}+m-k\right)}{1 \cdot 2 \cdot(2 m+1)(2 m+2)} z^{2}+\ldots\right] \\
& P(z)=z^{\ddagger-m} e^{-\frac{1 z}{z}}\left[1+\frac{\frac{1}{2}-m-k}{1 \cdot(-2 m+1)} z+\frac{\left(\frac{1}{2}-m-k\right)\left(\frac{3}{2}-m-k\right)}{1.2 \cdot(-2 m+1)(-2 m+2)} z^{2}+\ldots\right] \\
& =z^{\frac{b}{2}-m} e^{\frac{1}{2} z}\left[1-\frac{\frac{1}{2}-m+k}{1 \cdot(-2 m+1)} z+\frac{\left(\frac{1}{2}-m+k\right)\left(\frac{3}{2}-m+k\right)}{1 \cdot 2 \cdot(-2 m+1)(-2 m+2)} z^{2}-\ldots\right]
\end{aligned}
$$

(see Whittaker, Proc. Edin. Math. Soc., Vol. 32, p. 73).

From these equations it is obvious that when $-\frac{1}{2}<m<+\frac{1}{2}$, $W_{k, m}(z)$ will have a root when $z=0$, thus accounting for the root common to $W_{k, m}(z)$ and $W_{k-1, m}(z)$, mentioned in $\S 3$.

The graphs for the values $(k=-0 \cdot 1, m=0 \cdot 2),(k=-1 \cdot 1$, $m=0 \cdot 2)$, and $(k=-2 \cdot 1, m=0 \cdot 2)$ are of interest as indicating that when $k \rightarrow-\infty, W_{k, m}(z)$ tends to coincide with the $z$-axis.

\section{$\$ 5$. Roots of allied functions.}

By giving special values to $k$ and $m$ one can readily determine the disposition of the roots of certain well-known functions which can be expressed in terms of the function $W_{x, m}(z)$.

\section{The parabolic cylinder functions.}

These functions-denoted by $D_{n}(z)$-are connected with the functions $W_{k, m}(z)$ by means of the equation

$$
\text { zt } D_{2 k-\frac{1}{2}}(\sqrt{2 z})=2^{k-1} W_{k, t}(z) \text {. }
$$

Here, $m$ being positive, we need only consider the upper half of the plane in Fig. 1. The line for the boundary of no roots is $k-\frac{1}{2}-m=0$, i.e. $n-1=0$, for one root $k-\frac{3}{2}-m=0$, i.e. $n-3=0$, for two roots $k-\frac{5}{2}-m=0$, i.e. $n-5=0$, and so on. Hence, when $0<n<1, D_{n}(z)$ has no positive roots, when $1<n<3 D_{n}(z)$ has 
one positive root, when $3<n<5, D_{n}(z)$ has two positive roots, and so on. But, from $\S 6$, the number of roots is equal to $n$ if $n$ be an integer, or equal to the first integer greater than $n$ if $n$ be fractional. Hence the disposition of the roots in the case of the parabolic cylinder functions may be indicated in the following manner. If $n$ be an even integer, $=2 p$, say, there are $p$ positive and $p$ negative roots. If $n$ be odd and equal to $2 p+1$, there are $p$ positive roots, $p$ negative, and one zero root. If $n$ be equal to $2 p-e$, where $0<e<1$, there are $p$ positive and $p$ negative roots. Finally if $n$ be equal to $2 p+e$, there are $p$ positive and $p+1$ negative roots.

\section{Abel's function $\phi_{n}(z)$.}

This function which is discussed more fully in $\S 9$ is connected with the functions $W_{k, m}(z)$ by the relation

$$
\phi_{n}(z)=\frac{(-1)^{n}}{n !} z^{-\frac{1}{2}} e^{\frac{1}{z} z} W_{n+1,0}(z) .
$$

By referring to Fig. 1 and making $k=n+\frac{1}{2}, m=0$, one obviously at once deduces that the number of positive roots in $\phi_{n}(z)$ is equal to $n$.

\section{\$6. Theorem on rools of parabolic cylinder functions.}

A similar theorem on the alternation of zeros as established in $\$ 3$ obtains in the case of the parabolic cylinder functions. The differential equation satisfied by $D_{n}(z)$ may be written in the form

$$
\frac{d^{2} D_{n}}{d z^{2}}+\left(n+\frac{1}{2}-\frac{1}{4} z^{2}\right) D_{n}=0 \text {. }
$$

If we write

$$
D_{n}=e^{-1 z^{2}} v
$$

this equation is transformed into

$$
\frac{d^{2} v}{d z^{2}}-z \frac{d v}{d z}+n v=0 .
$$

Reasoning exactly similar to that employed in the case of the $W_{k, m}$-functions establishes the fact that the roots of $\frac{d v}{d z}$ and of $n v$ 


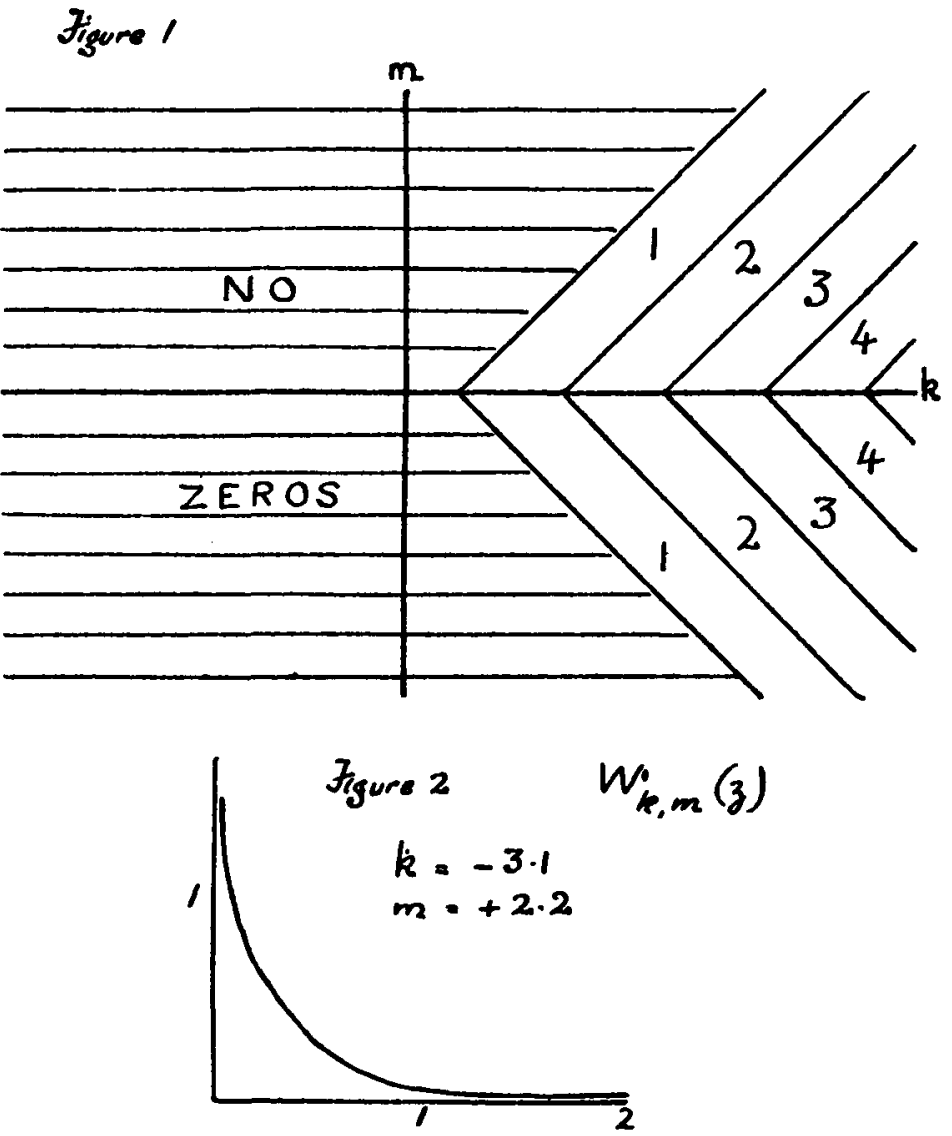

Igure 3

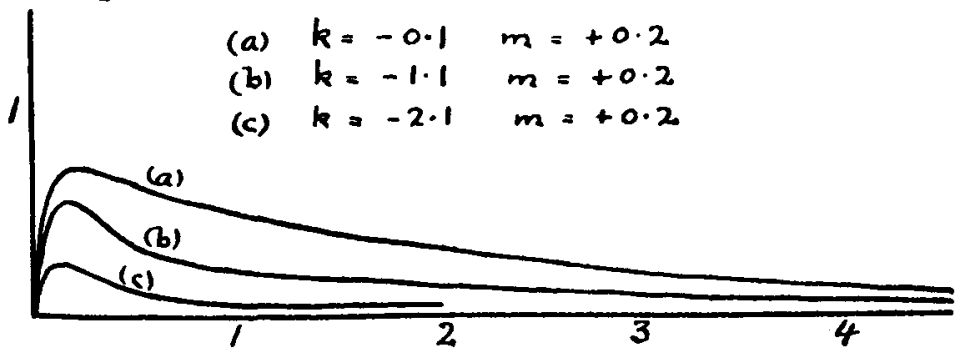



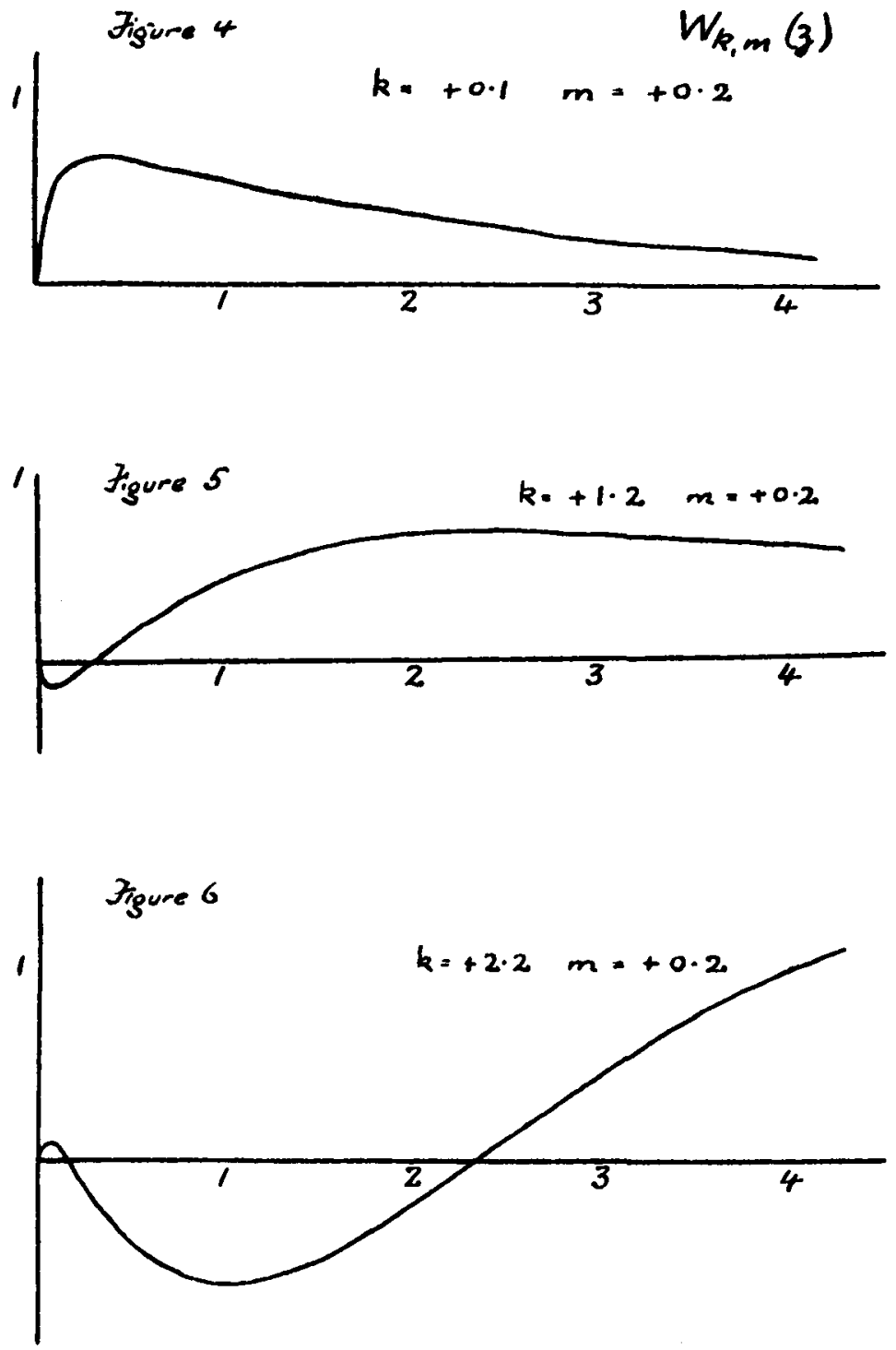





57

Figure 8 $D_{n}(z)$

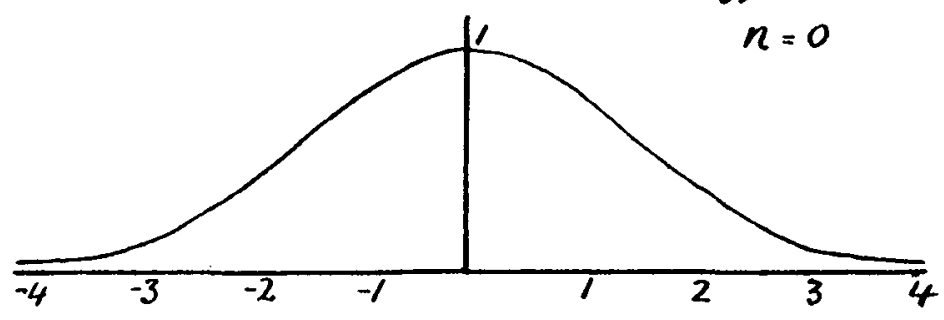

Figure 9
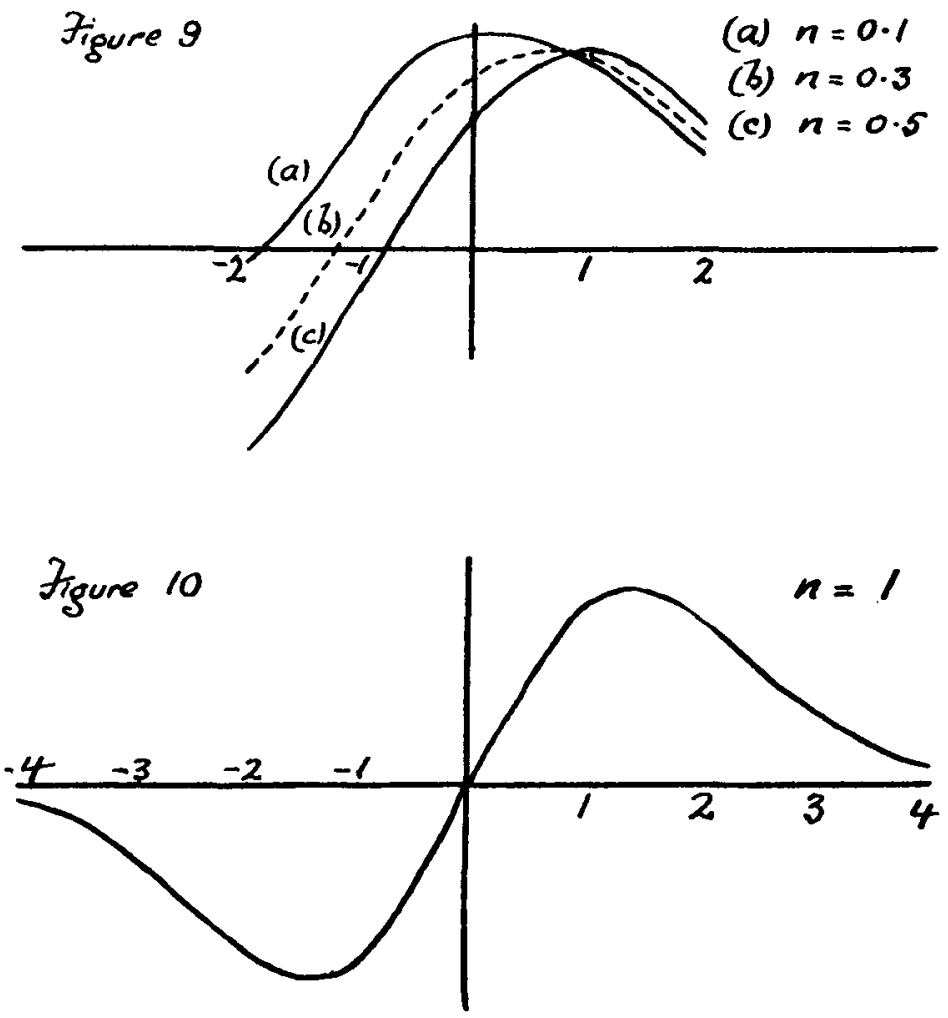

5 Vol. 38

https://doi.org/10.1017/S0013091500002352 Published online by Cambridge University Press 
occur alternately. This gives at once that the roots of $\frac{d D_{n}}{d z}+\frac{1}{2} z D_{n}$ and of $n D_{n}$ alternate.

Using the recurrence-formula

$$
\frac{d D_{n}}{d z}+\frac{1}{2} z D_{n}-n D_{n-1}=0
$$

we get that the zeros of $D_{n}(z)$ alternate with the zeros of $D_{n-1}(z)$.

Since $D_{0}(z)=e^{-\frac{1 z^{2}}{}}, D_{1}(z)=z e^{-\frac{1 z^{2}}{2}}$ and generally

$$
D_{n}(z)=(-1)^{n} e^{j z^{z}} \frac{d^{n}}{d z^{n}}\left(e^{-j z^{2}}\right)
$$

where $n$ is a positive integer, we see that $D_{0}(z)$ has no finite roots, and that every time $n$ is increased by unity a new root appears. Let now $n$ be a positive fraction such that $0<n<1$. Now

$$
D_{n}(z)=\frac{2^{\frac{n}{2}} \sqrt{\pi}}{\Gamma\left(\frac{1-n}{2}\right)} E_{n}(z)-\frac{2^{\frac{n+1}{2}} \sqrt{\pi}}{\Gamma\left(-\frac{n}{2}\right)} O_{n}(z)
$$

(See Proc. Edin. Math. Soc., Vol. 32, p. 7)

where

$$
E_{n}(z)=e^{-t z^{2}}\left[1-\frac{n}{2 !} z^{2}+\frac{n(n-2)}{4 !} z^{4}-\frac{n(n-2)(n-4)}{6 !} z^{6}+\ldots .\right]
$$

and

$$
O_{n}(z)=e^{-1 z^{2}}\left[z-\frac{n-1}{3 !} z^{3}+\frac{(n-1)(n-3)}{5 !} z^{5}-\ldots\right] .
$$

Since $e^{-1 z^{2}}$ does not vanish for any finite value of $z$, the appearance of the zeros will depend upon the series in the above equations.

When $0<n<1$, the series in $E_{n}(z)$ becomes

$$
1-\frac{n}{2 !} z^{2}-\frac{n(2-n)}{4 !} z^{4}-\frac{n(2-n)(4-n)}{6 !} z^{5}-\ldots
$$

and this is less than $1-\frac{n}{2 !} z^{2}+\frac{n^{2}}{4 !} z^{4}-\frac{n^{3}}{6 !} z^{6}+\ldots$, i.e. $<\cos \sqrt{n} z$, so long as $z$ is positive. 
Similarly the series in $O_{n}(z)$, viz.,

$$
z+\frac{1-n}{3 !} z^{3}+\frac{(1-n)(3-n)}{5 !} z^{5}+\ldots
$$

is greater than $\frac{1}{\sqrt{n}} \sin \sqrt{n z}$ for positive values of $z$. Hence as $z$ increases from 0 to 1 , the series in $E_{n}(z)$ steadily decreases from 1 to 0 , which latter value it attains at some point $\varepsilon<\frac{\pi}{2 \sqrt{n}}$. Also in the same interval the series in $O_{n}(z)$ increases from zero. Hence at some point $z<\frac{\pi}{2 \sqrt{n}}$ the two series will be equal. But $E_{n}(z)$ is an even function and $O_{n}(z)$ is an odd function. Therefore, when $0<n<1$, at some negative point $|z|<\frac{\pi}{2 \sqrt{n}}$ the two series will be equal but opposite in sign, and we shall have a zero value of $D_{n}(z)$. A closer approximation to the limit of the root may be obtained from the identity connecting $D_{n}(z), E_{n}(z), O_{n}(z)$ when it may readily be established that when $0<n<1$ we get a root in $D_{n}(z)$ at some point $|z|<\frac{1}{\sqrt{n}} \tan ^{-1} \sqrt{\frac{n}{2}} \frac{\Gamma\left(-\frac{n}{2}\right)}{\Gamma\left(\frac{1-n}{2}\right)}$.

The behaviour of the roots will be seen from Figs. 8-10. When $n=0$ there is no zero value of $D_{n}(z)$; but when $0<n<1$, a negative root appears, which passes to the right along the $z$-axis as $n$ increases to +1 , until, when $n=1, D_{n}(z)$ vanishes when $z=0$. A small increase in the value of $n$ introduces a new negative root, and as $n$ increases to +2 , the two roots now both increase until when $n=2$ they are equal and opposite in sign. This process goes on, every time $n$ exceeds an integer a new root appears, and as $n$ increases, the roots increase, and pass to the right along the $z$-axis.

\section{\$7. Functions related to $W_{k, m}(z)$.}

In his memoir on the functions $W_{k m}(z)$ Whittaker mentioned that there are various members of the family which have not yet been noticed, but which give promise of interesting properties, and be specially mentioned those families for which $m=0$ or $\frac{1}{2}$. 
An interesting class of functions is defined by

$$
\psi_{n}(z)=(-1)^{n} e^{k z} \frac{d^{n}}{d z^{n}}\left(e^{-k z} z^{n}\right)
$$

From the recurrence-formulae connecting these functions (see $\S 8$ ), one may readily derive

$$
z \frac{d^{2} y}{d z^{2}}+(1-k z) \frac{d y}{d z}+n k y=0
$$

as the linear differential equation satisfied by $\psi_{n}(z)$.

If in this equation we change the dependent variable by means of the substitution

$$
y=e^{\jmath k z} z^{-1} u,
$$

the equation is obtained

$$
\frac{d^{2} u}{d z^{2}}+u\left[-\frac{k^{2}}{4}+\frac{\frac{k}{2}+n k}{z}+\frac{\frac{1}{4}}{z^{2}}\right]=0,
$$

or writing $k z$ for $z$

$$
\frac{d^{2} u}{d z^{3}}+u\left[-\frac{1}{4}+\frac{n+\frac{1}{2}}{z}+\frac{\frac{1}{4}}{z^{2}}\right]=0
$$

which is obviously the linear differential equation of $W_{n+1,0}(z)$. Hence the function $\psi_{n}(z)=$ const. $\times e^{\frac{1}{2} z} z^{-\frac{1}{2} W_{n+\frac{1}{2}, 0}}(z)$.

\$8. Properties of $\psi_{n}(z)$.

1. Expansion as series. - If we consider the equation

$$
z \frac{d^{2} y}{d z^{2}}+(1-k z) \frac{d y}{d z}+n k y=0
$$

and try to get an expansion valid in the neighbourhood of the origin, we find that the exponents of the indicial equation are zero, and we get in the ordinary way

$$
y(z)=1-n k z+\frac{n(n-1)}{(2 !)^{2}} k^{2} z^{2}-\frac{n(n-1)(n-2)}{(3 !)^{2}} k^{3} z^{y}+\ldots
$$

Now $\psi_{n}(z)=(-1)^{n}(D-k)^{n} z^{n}$

$$
=(-1)^{n} n !\left[1-n k z+\frac{n(n-1)}{(2 !)^{2}} k^{2} z^{2}-\frac{n(n-1)(n-2)}{(3 !)} k^{3} z^{3}+\ldots\right]
$$

so that $\psi_{n}(z)=(-1)^{n} n ! y(z)$. 
2. Asymptotic expansions. - An asymptotic expansion for $\psi_{n}(z)$ may readily be found from the linear differential equation by assuming as solution an expression of the form

$$
z^{r}\left(1+\frac{a}{z}+\frac{b}{z^{2}}+\ldots\right)
$$

when we get

$$
\psi_{n}(z)=z^{n}\left[1-\frac{n^{2}}{k z}+\frac{n^{2}(n-1)^{2}}{2 ! k^{2} z^{2}}-\frac{n^{2}(n-1)^{2}(n-2)^{2}}{3 ! k^{3} z^{3}}+\ldots\right]
$$

Again, if in the differential equation we substitute $u=e^{-k z} y$, we obtain the series

$$
y=e^{k z} z^{-n-1}\left[1+\frac{(n+1)^{2}}{k z}+\frac{(n+1)^{2}(n+2)^{2}}{2 ! k^{2} z^{2}}+\frac{(n+1)^{2}(n+2)^{2}(n+3)^{2}}{3 ! k^{3} z^{3}}+\ldots\right]
$$

which is another asymptotic expansion of $\psi_{n}(z)$.

3. Recurrence formulae.-Using Cauchy's formula we have the identity

$$
\frac{(-1)^{n} 2 \pi i}{n !} e^{-k z} \psi_{n}(z)=\int_{\delta} e^{-k x} x^{n}(x-z)^{-n-1} d x .
$$

By means of this identity we obtain, on integrating the righthand side by parts, the recurrence-formula

$$
\psi_{n}(z)+\{(2 n-1)-k z\} \psi_{n-1}(z)+(n-1)^{2} \psi_{n-2}(z)=0 .
$$

Again, by differentiation of the same identity, we obtain

$$
z \frac{d \psi_{n}(z)}{d z}-n \psi_{n}(z)-n^{2} \psi_{n-1}(z)=0 .
$$

From these two formulae can be derived

$$
\begin{aligned}
& \text { (i) }(k z-n) \frac{d \psi_{n}(z)}{d z}-n^{2} \frac{d \psi_{n-1}(z)}{d z}-n k \psi_{n}(z)=0 \\
& \text { (ii) } \frac{d \psi_{n}(z)}{d z}+n \frac{d \psi_{n-1}(z)}{d z}-n k \psi_{n-1}(z)=0 .
\end{aligned}
$$

4. Orthogonal nature of the functions $\psi_{n}(z)$.

Consider $\int_{0}^{\infty} e^{-k x} \psi_{m}(z) \psi_{n}(z) d z$ where $n \neq m, n$, say, being the greater. 
This integral may be written $\int_{0}^{\infty} \frac{d^{n}}{d z^{n}}\left(e^{-k z} z^{n}\right) \psi_{m}(z) d z$, which, on integrating by parts, is equal to

$$
\int_{0}^{\infty} e^{-k z} z^{n} \frac{d^{n}}{d z^{n}} \psi_{m}(z) d z .
$$

Now the highest term in $\psi_{m}(z)$ is $k^{m} z^{m}$, and $n>m$,

$$
\therefore \quad \int_{0}^{\infty} e^{-k z} \psi_{m}(z) \psi_{n}(z) d z=0
$$

By similar reasoning it may be shewn that

$$
\left.\int_{0}^{\infty} e^{-k z} \overline{\psi_{n}(z)}\right|^{2} d z=\frac{(n !)^{2}}{k}
$$

If we seek to express $x^{\mu}$ in the form

$$
x^{\mu}=a_{0} \psi_{0}(x)+a_{1} \psi_{1}(x)+\ldots+a_{\mu} \psi_{\mu}(x),
$$

we get, on multiplying both sides by $e^{-k x} \psi(x)$ and integrating from 0 to $\infty$,

$$
\begin{aligned}
& \frac{(m !)^{2}}{k} a_{m}=\int_{0}^{\infty} e^{-k x} x^{\mu} \psi_{m}(x) d x \\
& =(-1)^{m} m ! \int_{0}^{\infty} e^{-k x} x^{\mu}\left[1-{ }_{m} C_{1} k x+{ }_{m} C_{2} \frac{(k x)^{2}}{2 !}-\ldots+(-1)^{m} \frac{(k x)^{m}}{m !}\right] d x \\
& =\frac{(-1)^{m} m ! \Gamma(\mu+1)}{k^{\mu}}\left[1-{ }_{m} C_{1}(\mu+1)+{ }_{m} C_{2} \frac{(\mu+2)}{1 \cdot 2} \frac{(\mu+1)}{2}-\ldots \ldots\right. \\
& \left.\ldots \ldots+(-1)^{m} \frac{(\mu+m)(\mu+m-1) \ldots(\mu+1)}{1.2 .3 \ldots m}\right] .
\end{aligned}
$$

But by the Remainder Theorem, the part inside the square brackets is equal to

$$
\begin{gathered}
\frac{(-1)^{m}}{m !} \mu(\mu-1) \ldots(\mu-m+1) \\
=\frac{(-1)^{m}}{m !} \frac{\mu !}{(\mu-m) !} .
\end{gathered}
$$

Hence finally we obtain $a_{m}=\frac{\{\Gamma(\mu+1)\}^{2}}{k^{\mu}\{\Gamma(m+1)\}^{2} \Gamma(\mu-m+1)}$. 
5. Second solution of the differential equation.-We have already seen that $\psi_{n}(z)=(-1)^{n} e^{k x} \frac{d^{n}}{d z}\left(e^{-k z} z^{n}\right)$ is a solution of the differential equation

$$
z \frac{d^{2} y}{d z^{2}}+(1-k z) \frac{d y}{d z}+n k y=0 .
$$

If in this equation we make the substitution $y=e^{x t} u$ we get the equation

$$
z \frac{d^{2} u}{d z^{2}}+(1+k z) \frac{d u}{d z}+(n+1) k u=0
$$

of which a solution will obviously be obtained by replacing $n$ by $-n-1$ and $k$ by $-k$ in $\psi_{n}(z)$.

Doing so, we find that when $n$ is a negative integer, another solution of the differential equation is given by

$$
(-1)^{n+1} \frac{d^{-n-1}}{d z^{-n-1}}\left(e^{k z} z^{-n-1}\right) \text {. }
$$

\$9. Abel': ф-function.

Another set of functions associated with $W_{k, m}(z)$ when $m=0$ is Abel's $\phi$-function.* In his Civures Completes (1881 edition), Vol. 2, p. 284, he defines $\phi_{m}(z)$ as the coefficient of $v^{m}$ in the expansion of

$$
\frac{1}{1-v} e^{-\frac{z v}{1-v}} \text {. }
$$

He obtains the expansion

$$
\phi_{m}(z)=1-m z+\frac{m(m-1)}{1.2} \frac{z^{2}}{2 !}-\ldots \mp \frac{z^{m}}{m !},
$$

shews that $\int_{0}^{\infty} e^{-z} \phi_{m}(z) \phi_{n}(z) d z=1$ or 0 according as $m=n$ or $m \neq n$, and finally proves that if $x^{\mu}$ be expanded in the form

then

$$
\begin{gathered}
x^{\mu}=A_{0} \phi_{0}(x)+A_{1} \phi_{1}(x)+\ldots+A_{\mu} \phi_{\mu}(x) \\
A_{m}=(-1)^{m} \frac{\Gamma(\mu+1) \Gamma(\mu+1)}{\Gamma(m+1) \Gamma(\mu-m+1)} .
\end{gathered}
$$

This function $\phi(z)$ is really a particular case of the function $\psi(x)$ already considered, for if we make $k=1$ in the latter, and

- The connexion of the $\phi$-function with the $W_{k m}$-function was first noticed by $\mathbf{H}$. Bateman. 
compare the expansion of this with the expansion of $\phi(z)$ given above, we at once find that

$$
\psi_{n}(z)=(-1)^{n} n ! \phi_{n}(z) .
$$

Hence it at once follows that $\phi_{n}(z)$ belongs to the same group of the functions $W_{k, m}(z)$ that $\psi_{n}(z)$ does, since we at once have the relation

$$
\phi_{n}(z)=\text { const. } \times e^{\frac{1 z}{2} z} z^{-\frac{1}{1}} W_{n+\frac{1}{2}, 0}(z) .
$$

The properties of $\phi_{n}(z)$ quoted above at once follow from the corresponding properties of $\psi_{n}(z)$ already given by putting in the latter $k=1$ and noting the relation connecting $\phi_{n}(z)$ and $\psi_{x}(z)$. In addition, other properties of $\phi_{n}(z)$ may be obtained. Thus its linear differential equation is

$$
z \frac{d^{2} y}{d z^{2}}+(1-z) \frac{d y}{d z}+n y=0
$$

while its asymptotic expansion, etc., may be derived in a similar manner. 